\title{
Carbon nanoparticles guide contralateral central neck dissection in patients with papillary thyroid cancer
}

\author{
WENBIN YU, GUOHUI XU, JUNYONG SUN and NAISONG ZHONG \\ Key Laboratory of Carcinogenesis and Translational Research, Department of Head and Neck \\ Surgery, Peking University Cancer Hospital and Institute, Beijing 100142, P.R. China
}

Received March 17, 2016; Accepted December 22, 2017

DOI: $10.3892 / \mathrm{ol} .2018 .8691$

\begin{abstract}
The treatment of contralateral central neck lymph node metastasis is controversial in patients with papillary thyroid cancer. The present study reports the use of carbon nanoparticles (CNs) as lymph node tracers and discusses the potential role of predicting contralateral central neck metastasis is evaluated, so as to guide contralateral central neck dissection (CND). A total of 70 consecutive patients with papillary thyroid cancer were enrolled in the present study. All patients underwent a total or near-total thyroidectomy plus bilateral CND, during which CNs were used as a lymph node tracer. Of the 70 enrolled patients, $51(72.86 \%)$ were confirmed to have lymph node metastasis in the central neck, $50(71.43 \%)$ patients in the ipsilateral central neck and $14(20.00 \%)$ in the contralateral central neck. A total of $579(84.90 \%)$ lymph nodes were stained black by CNs. Of the 193 metastatic lymph nodes, 168 were located in the ipsilateral central compartment and the other 25 in the contralateral central compartment. A total of 147 (76.17\%) metastatic lymph nodes were stained black. A total of 21 metastatic lymph nodes were found in the contralateral central compartment, 4 metastatic lymph nodes of contralateral central compartment were not black-stained. The sensitivity and specificity of CNs for contralateral metastasis was 84 and $25 \%$, respectively. Contralateral central lymph node metastasis was significantly associated with extrathyroid extension and the presence of ipsilateral central neck lymph node metastasis. Together, the results of the present study reveal that $\mathrm{CNs}$ might accurately predict contralateral central lymph nodes metastasis and could be used to direct CND.
\end{abstract}

\section{Introduction}

Papillary thyroid cancer (PTC) is the most common subtype of thyroid cancer, accounting for more than $90 \%$ of all

Correspondence to: Dr Wenbin Yu, Key Laboratory of Carcinogenesis and Translational Research, Department of Head and Neck Surgery, Peking University Cancer Hospital and Institute, 52 Fucheng Road, Haidian, Beijing 100142, P.R. China

E-mail: yuwenbin429@qq.com

Key words: carbon nanoparticle, central, papillary, thyroid cancer cases (1). The incidence of PTC has been increasing rapidly in recent years, largely because of advances in early detection using ultrasonography and fine-needle aspiration biopsy $(1,2)$. Cervical lymph node metastases are common in patients with PTC, in whom the reported metastatic rate is $30-80 \%$, with the central neck being the most common site $(3,4)$. Recurrence following treatment is common, occurring in up to $30 \%$ of all patients and $75 \%$ of local recurrence occurs in the central neck; it can occur up to 20 years after the initial diagnosis $(5,6)$, Therapeutic central neck dissection (CND) is routinely performed in patients with PTC and clinically positive lymph nodes $(7,8)$. Although the use of prophylactic CND in patients with clinically negative lymph nodes remains controversial because of its potential morbidity and unclear benefit $(9,10)$, many clinicians continue to recommend prophylactic CND owing of the high rate of central lymph node metastasis; however, up to $80 \%$ of positive lymph node are undetectable, microscopic metastatic lymph node disease $(11,12)$. Prophylactic CND can remove microscopic disease in central lymph nodes, which is unlikely to be eradicated by radioactive iodine therapy (13). Evidence indicates that prophylactic CND may prevent recurrence and improve overall survival (14). Reoperation for recurrent PTC in the central lymph node has a high rate of complications, including, hypoparathyroidism and recurrent laryngeal nerve palsy (15). Prophylactic CND can permit accurate nodal staging [one-third of patients increased to stage III disease due to lymph node metastasis detected by prophylactic CND (16)] and provide information about prognosis and guidelines for treatments such as radioactive iodine therapy and hormone suppression therapy (17). Routine prophylactic CND has been recommended by the Japanese Society of Thyroid Surgeons and the French Society of Otolaryngology Head and Neck Surgery (18).

Controversy remains regarding the extent of prophylactic CND in unilateral PTC (unilateral vs. bilateral CND). Although the majority of metastatic lymph nodes are located in the ipsilateral central neck, the metastatic rate of bilateral central neck was reported to be $18.9-30.6 \%$ (19). Owing to the potential higher risks of complications for bilateral CND and the lower metastatic rate of contralateral central neck, efforts have been made to predict contralateral central neck metastasis using clinicopathological characteristics (8). As a novel lymphatic tracer, the use of carbon nanoparticles (CNs) have been reported to efficiently guide CND (20). The present study 
reports the use of CNs on predicting contralateral central neck metastasis and evaluates its clinical value.

\section{Materials and methods}

Imaging agent for lymphatic vessels. CNs (China Food and Drug Administration approval no. H20041829; Chonqing Lummy Pharmaceutical Co., Ltd., Chongqing, China) can be injected as a suspension $(50 \mathrm{mg} / \mathrm{ml})(21,22)$. This product is a stable suspension of CNs $150 \mathrm{~nm}$ in diameter. The diameter of the CNs is greater than the capillary endothelial cell gap $(20-50 \mathrm{~nm})$ and smaller than the lymphatic capillary endothelial cell gap (120-500 nm), so a small proportion of the CNs may be captured by macrophages, then enter the lymphatic duct but not the blood circulation. There have been no reports of CNs exerting toxic side effects in humans $(23,24)$.

Patients. A total of 70 consecutive patients were enrolled into this study between January 2012 and June 2013; all patients were diagnosed with PTC by preoperative aspiration pathology and underwent the initial surgery in the Department of Head and Neck Surgery, Peking University Cancer Hospital \& Institute (Beijing, China), all the tumors were located in one lobe. The inclusion criterion was PTC (size of lesion, 1-4 cm). Exclusion criteria included non-thyroid cancer, previous thyroid or parathyroid surgery, preoperative hypoparathyroidism or hypocalcemia, pregnancy or lactation, surgery confirming suspicion of the presence of lateral neck lymph node metastasis, age $<18$ years, and an inability to comply with the follow-up protocol. In total, 14 patients were males and 56 were female, with a male to female ratio of 1:4. The ages of patients ranged between 19 and 75 years, with a median of 45.23 years. All surgery was performed by the same medical team, and all the patients underwent total or near-total thyroidectomy plus bilateral CND. Approval was obtained from the Ethics Committee of Beijing University Cancer Hospital prior to these procedures; all patients provided informed consent for inclusion in the present study.

Surgical procedure. All patients underwent general anesthesia intubation, and were placed supinely with necks hyperextended. A transverse incision of $\sim 5 \mathrm{~cm}$ was performed in a skin crease $2 \mathrm{~cm}$ above the sternal notch. The skin, subcutaneous tissue, and platysma were cut layer by layer. Flap separation was performed under the platysma before making a longitudinal incision in the linea alba cervicalis. The strap muscles retracted and the front ipsilateral thyroid was revealed; the side and rear parts of the lobe were kept intact to reduce damage to the lymphatic network around the thyroid. In the $\mathrm{CN}$ group, $0.1 \mathrm{ml}$ of carbon nanoparticles per point was injected into tissues surrounding the tumor using a skin test syringe. In total, 2 or 3 spots were injected for each tumor, with the total amount injected being no more than $0.5 \mathrm{ml}$ per lobe. Prior to injection, the $\mathrm{CN}$ suspension was inserted deeply and aspirated to ensure it would not be injected into the blood vessels. The CNs suspension was slowly injected into the thyroid tissues. Upon completion, gentle pressure was applied on the needle puncture site to prevent solution leakage.

After 5 min, the lymphatic ducts and the lymph nodes became black-stained (Fig. 1). Total thyroidectomy or near-total thyroidectomy was then performed according to the meticulous capsular dissection technique. Node clearance of the central neck compartment was performed cranially to the two superior thyroid arteries and the pyramidal lobe, caudally to the innominate vein, laterally to the carotid sheaths, and dorsally to the prevertebral fascia (25). When the thyroidectomy was completed, the tumors were sent for routine frozen pathology examination to confirm the preoperative aspiration pathological diagnosis. Ipsilateral CND included the removal of prelaryngeal, pretracheal and paratracheal nodes on the side of the tumor. The resected specimens of lymph nodes were carefully checked and labeled as being either black-stained or non-stained in the $\mathrm{CN}$ group. All metastatic lymph node specimens were sent for pathological examination. Specimens were checked for the presence of the parathyroid, for unintentionally removed parathyroid glands, frozen section biopsy of one-fourth to one-third of the removed parathyroid glands was performed during surgery for pathological confirmation. When confirmed to be the parathyroid gland, the remaining tissue was transplanted into the sternocleidomastoid muscle.

Surveillance and management of postoperative complications. Serum calcium was assessed at 6:00 am on the first postoperative day, postoperative hypocalcemia was defined as a calcium level of $<2.0 \mathrm{mmol} / 1$ (reference range, 2.10-2.60 mmol/l), with or without clinical symptoms of hypocalcemia or subnormal serum calcium levels $(2.0-2.1 \mathrm{mmol} / \mathrm{l})$ with neuromuscular symptoms. Calcium supplementation was not routinely administered to patients. Calcium and vitamin D were routinely prescribed to patients with symptomatic hypocalcemia, Intravenous substitution of calcium was not routine unless serious symptomatic hypocalcemia was present. Vocal cord palsy, as confirmed by laryngoscopy, was considered permanent if it lasted for $>6$ months.

Statistical analysis. The Student t-test and $\chi^{2}$ test were used for the measurement and count data, respectively. SPSS 17.0 (SPSS, Inc., Chicago, IL, USA) was used to perform statistical analysis; $\mathrm{P}<0.05$ was considered to indicate a statistically significant difference.

\section{Results}

General information of patients. The clinicopathological characteristics of the patients are summarized in Table I. All the tumors were located in one lobe. All patients underwent total or near-total thyroidectomy plus bilateral CND. Papillary thyroid cancer was confirmed by perioperative and postoperative pathology for all patients. The mean age of all the patients was $45.49 \pm 18.97$ years (range, 19-75 years). Of the patients enrolled, $52.86 \%$ (37/70) were $<45$ years of age, and $47.14 \%$ $(33 / 70)$ were $\geq 45$ years of age. A total of 56 women $(80.00 \%)$ and 14 men $(20.00 \%)$ participated. The mean size of the primary tumor was $1.98 \pm 0.72 \mathrm{~cm}$. All patients had a primary tumor $\geq 1 \mathrm{~cm}$, multifocal tumors were observed in 16 patients (22.86\%). Extrathyroid extension was observed in 28 patients (40.00\%).

Central lymph node metastasis. The central lymph node metastasis status of the patients is summarized in Table II. Of 
Table I. The clinical pathological characteristics of the patients.

\begin{tabular}{lc}
\hline Clinicopathological characteristics & Value \\
\hline Age, years $^{\mathrm{a}}$ & $45.49 \pm 18.97$ \\
$<45$ & $37(52.86)$ \\
$\geq 45$ & $33(47.14)$ \\
Sex, $\mathrm{n}$ & \\
Male & 14 \\
Female & 56 \\
Size of primary tumor, $\mathrm{cm}^{\mathrm{a}}$ & $1.98 \pm 0.72$ \\
Extrathyroid extention, $\mathrm{n}(\%)$ & $28(40.00)$ \\
Multifocal tumor, $\mathrm{n}(\%)$ & $16(22.86)$ \\
\hline
\end{tabular}

${ }^{\mathrm{a}}$ Mean \pm standard deviation.

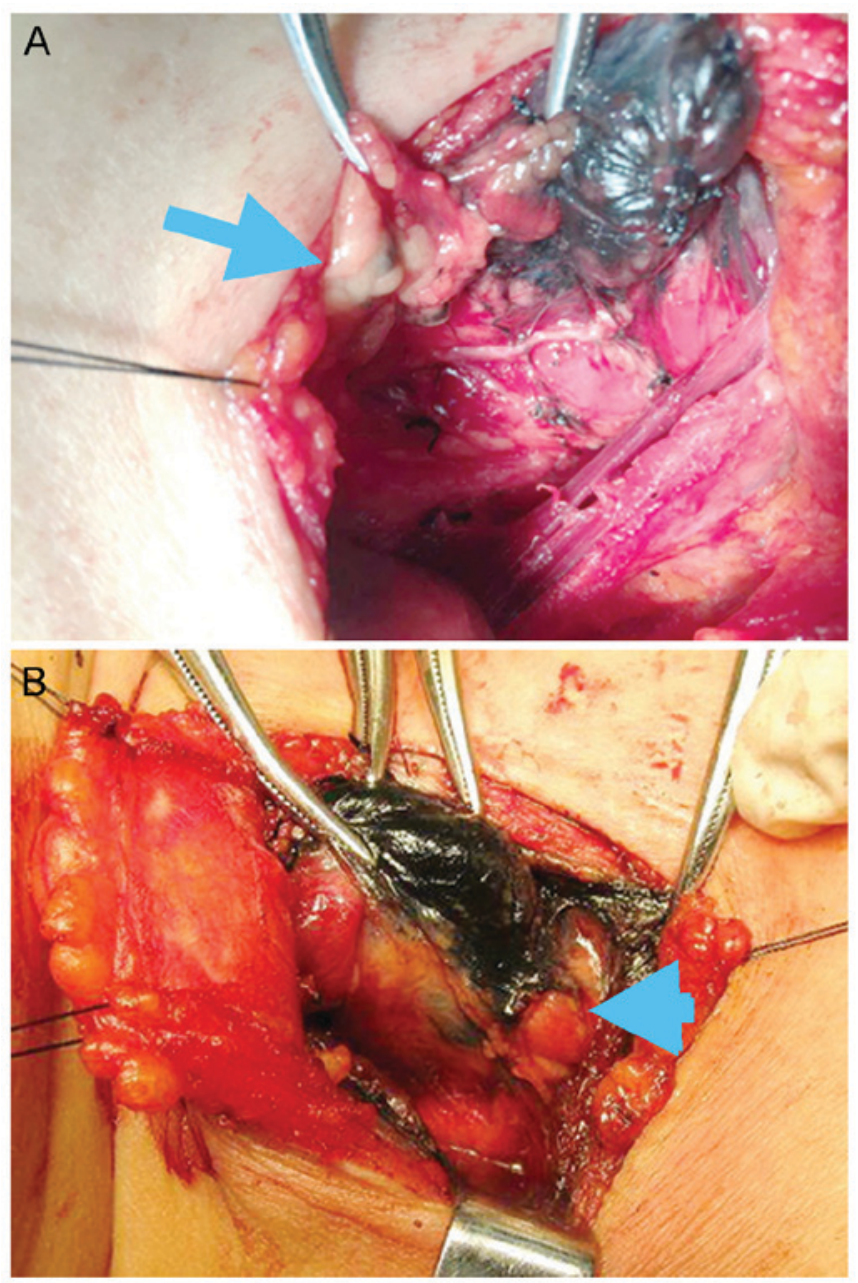

Figure 1. (A) Black-stained lymph node (arrow). (B) Non-black-stained parathyroid in contrast to black-stained thyroid gland (arrow).

the enrolled 70 patients, 51 patients $(72.86 \%)$ were confirmed to have lymph node metastasis in the central neck. A total of $50(71.43 \%)$ patients had lymph node positive for metastasis in the ipsilateral central neck, which included the prelaryngeal, pretracheal and paratracheal nodes on the side of the tumor. By contrast, 14 (20.00\%) patients had contralateral central neck lymph node metastases, with only $1(1.43 \%)$ exhibiting contralateral metastasis but not the ipsilateral central compartment metastasis (skip metastasis). A total of 682 lymph nodes were found in this group, with an average of $9.74 \pm 4.33$ per patient (range, $4-22$ pieces). The diameter of 279 (40.91\%) lymph nodes was $<5 \mathrm{~mm}$. Among the 682 detected lymph nodes, 579 (84.90\%) were black-stained by CNs. Of the 193 metastatic lymph nodes, 168 were located in ipsilateral central compartment and the other 25 in the contralateral central compartment. Of the 193 metastatic lymph nodes, 147 (76.17\%) were black-stained and the other 46 (23.83\%) were not. A total of 63 metastatic lymph nodes were found that were $<5 \mathrm{~mm}$ in the metastatic black-stained lymph nodes. A total of 128 lymph nodes were stained black in the ipsilateral central compartment and 21 in the contralateral central compartment; 4 metastatic lymph nodes in the contralateral central compartment were not stained black. The sensitivity and specificity of CNs for contralateral metastasis were 84 and $25 \%$, respectively. Statistical analysis of the clinicopathological factors associated with contralateral lymph nodes metastases (Table III) revealed that contralateral central lymph nodes metastasis was significantly associated with extrathyroid extension and the presence of ipsilateral central neck lymph node metastasis. By contrast, sex, presence of multifocality and age were not associated with contralateral lymph nodes metastasis. Of the 14 patients with contralateral central neck lymph node metastasis, 13 had nodes positive for metastasis in the ipsilateral central neck and 9 patients had extrathyroid extension.

Side effects and operative complications. No evident systemic toxicity occurred in these patients during and following surgery. Hoarseness occurred to two patients due to tumor adhesion. Notably, the parathyroid glands could be well protected during surgery as parathyroid glands could not be stained black, unlike the black-stained lymph nodes (Fig. 1), so could be easily distinguished and kept in situ. In total, 12 patients $(17.14 \%)$ were confirmed to be hypocalcemia. Pathological results revealed that 5 incidences of accidental parathyroid resection occurred; the resected parathyroid glands were re-implanted into sternocleidomastoid muscle, as described previously (26).

\section{Discussion}

Central neck lymph node metastasis is fairly common in papillary thyroid cancer and is observed in $20-90 \%$ of patients $(1,2,12)$. Therapeutic CND is routinely performed in patients with PTC with lymph nodes clinically positive for metastasis $(7,8)$. Although controversy exists regarding prophylactic CND in patients with clinically negative lymph nodes owing to its potential morbidity and unclear benefit $(9,10)$. At the time of writing, there was no effective means of predicting central neck lymph node metastasis. Ultrasound and computerized tomography are the most common methods used to preoperatively evaluate metastatic lymph nodes in PTC patients $(27,28)$. Ultrasound is a sensitive and specific test for detecting lateral cervical lymph node metastasis; however, it was not sufficiently sensitive in assessing central neck lymph nodes owing to the presence of multifocal thyroid nodules and surrounding structures in the central neck (27). Ultrasound 
Table II. Central neck metastasis of patients under the guidance of carbon nanoparticles.

Variables

Ipsilateral central neck, $\mathrm{n}(\%)$

Contralateral central neck, $\mathrm{n}$

$(\%)$

Patients with metastases
Dissected lymph nodes
Lymph nodes $<5 \mathrm{~mm}$
Black stained lymph nodes
Metastatic lymph nodes
Black-stained metastatic lymph nodes

Table III. Clinicopathological factors associated with contralateral central neck metastasis.

\begin{tabular}{lcc}
\hline & $\begin{array}{c}\text { Patients with central } \\
\text { neck contralateral } \\
\text { metastasis, n (\%) }\end{array}$ & P-value \\
\hline Age, years & $7(18.92)$ & \\
$<45$ & $7(21.21)$ & 0.811 \\
$\geq 45$ & & \\
Sex & $3(11.00)$ & \\
Male & $11(45.00)$ & 0.881 \\
Female & & \\
Extrathyroid extension & $9(32.14)$ & 0.038 \\
Yes & $5(11.90)$ & \\
No & & 0.569 \\
Multifocal tumor & $4(25.00)$ & \\
Yes & $10(18.52)$ & \\
No & & \\
Ipsilateral central neck & & \\
lymph node & & \\
Yes & $13(26.00)$ & \\
No & $1(5.26)$ & \\
\hline
\end{tabular}

has a sensitivity of $66.3 \%$ with a specificity of $88.4 \%$ (27). Computerized tomography has been found to have a sensitivity of $67 \%$ and a specificity of $79-91 \%$ (28). Positron emission tomography is not routinely preoperatively used in PTC patients as its sensitivity has been reported to be $<40 \%$ (29).

Microcarcinoma and tumors $>1 \mathrm{~cm}$ require different surgical treatment, as certain microcarcinomas may be treated without surgery (30), whilst tumors $>1 \mathrm{~cm}$ must be treated by surgery. A tumor size $>1 \mathrm{~cm}$ was reported to be an independent predictor of central metastasis and disease-free survival (25); as such, only patients with tumors $>1 \mathrm{~cm}$ were selected as subjects in the present study. In the study group, 51 patients (72.86\%) were confirmed to have lymph node metastasis in the central neck, 50 $(71.43 \%)$ in the ipsilateral central neck, and 14 (20.00\%) patients in the contralateral central neck. The results of the present study, in accordance with those of a previous study, indicate that ipsilateral central neck metastasis was the most frequently involved region in the central neck compartment (25). Skip metastasis is generally uncommon (31), and was only observed in one patient $(1.43 \%)$ in the present study, characterized by metastasis to the contralateral but not the ipsilateral central compartment. The results of the current study revealed that contralateral central lymph node metastasis was significantly associated with the presence of ipsilateral central neck lymph node metastasis, a finding that was also strongly supported by other studies $(9,25,32)$. Lim et al (33) demonstrated that ipsilateral central lymph node metastasis was the only independent predictor for the presence of contralateral central lymph node metastasis and suggested that elective contralateral CND should be considered when performing initial surgery for PTC with a tumor diameter of $>1 \mathrm{~cm}$ and the presence of ipsilateral central lymph node metastasis. The results of the present study also revealed that contralateral central lymph nodes metastasis was significantly associated with extrathyroid extension, which tallied with the findings of previous studies $(10,25)$, whereas extrathyroid extension was difficult to diagnose pre- or intra-operatively. Other factors associated with contralateral lymph nodes metastasis are male gender, lateral neck lymph nodes metastasis and tumor multifocality $(34,35)$. Among these clinicopathological factors, ipsilateral central lymph node metastasis was strongly indicated to be the predictive factors. Raffaelli et al (36) reported that routine ipsilateral CND plus frozen section examination of the ipsilateral nodes may represent a valid alternative to prophylactic bilateral CND. As the rate of metastasis to the ipsilateral central neck is high, many metastatic lymph nodes are too small to be detected by frozen section examination, meaning that this strategy may aid prediction of contralateral central lymph node metastasis.

Recently, a suspension of CNs has been used to aid the identification lymph nodes in lymph node dissection (20). This product is a stable suspension of CNs of $150 \mathrm{~nm}$ in diameter, which is greater than the capillary endothelial cell gap $(20-50 \mathrm{~nm})$ and smaller than the lymphatic capillary endothelial cell gap (120-500 nm). Following injection into the thyroid glands around the tumor, $\mathrm{CNs}$ are rapidly captured by macrophages. The particles then enter the lymphatic vessels and accumulate in the lymph nodes, staining them black. CNs have been successfully used in the detection of sentinel lymph nodes in breast and gastric cancer (21). Hao et al (37) used CNs as tracers for sentinel lymph node biopsy on patients with papillary thyroid microcarcinoma who underwent CND; the sensitivity, specificity, accuracy rate, and false negative rate were 93.3, 100, 97 and 5.2\%, respectively. The authors concluded that the CNs method better aided the screening 
and selection of patients who are most likely to benefit from cervical lymph node dissection (37). CNs stain the majority of lymph nodes, with the reported rate of $\mathrm{CN}$ staining was $69.89-95.26 \%(20,37,38)$, with the black-staining rate in the present study being $84.90 \%$. More lymph nodes $<5 \mathrm{~mm}$ were detected with the assistance of $\mathrm{CNs}$, which cannot be easily recognized without any assistance. The diameter of $279(40.91 \%)$ lymph nodes in the present study was $<5 \mathrm{~mm}$ Zhu et al (20) reported that significantly more small lymph nodes, particularly those $<2 \mathrm{~mm}$, were detected, compared with the control group for whom CNs were not used. A total of 63 metastatic lymph nodes were found that were $<5 \mathrm{~mm}$ in the metastatic black-stained lymph nodes in this study using CNs.

$\mathrm{CNs}$ have been demonstrated to detect more lymph nodes, particularly small lymph nodes in the present study. To the best of our knowledge, the present study evaluated the potential role of predicting contralateral central lymph node metastasis in PTC under the guidance of CNs for the first time. The results of the present study revealed that 21/25 (84\%) metastatic lymph nodes were stained black in the contralateral central compartment. the sensitivity and specificity of CNs for contralateral metastasis were 84 and $25 \%$, respectively. CNs may be useful to aid prediction of the presence of contralateral central lymph node metastasis; the majority of the metastatic lymph nodes were stained black, the black-stained lymph nodes may be resected for fast-frozen pathology to determine whether contralateral CND should be performed. Additionally, all the black stained lymph nodes may be resected (limited CND) instead of standard CND, so as to decrease the potential complications (such as hypoparathyroidism and recurrent laryngeal nerve palsy). Further studies could evaluate whether fast frozen pathology or limited CND is feasibility in prophylactic contralateral CND.

The present study found that, unlike the lymph nodes, parathyroid glands could not be stained black following CNs injection, as revealed in previous studies $(20,37)$. CNs cannot enter the blood circulation, meaning that parathyroid glands and the surrounding blood supply appear different from the black-stained lymph nodes. In total, 12 patients (17.14\%) were confirmed to be hypocalcemic in the present study, lower than the number found in a previous study (30.77\%) (39) (the patients in the two studies received the same treatment). Further study is warranted to evaluate the potential protective role of CNs to the parathyroid glands.

The results of the present study indicate that the use of CNs is safe, and that no evident systemic toxicity was observed in patients during and following surgery. These results suggest that CNs can effectively predict contralateral CND, which could have promise in clinical use.

\section{Acknowledgements}

The authors would like to thank Professor Wei Zhao from the Beijing Cancer Hospital for providing technical assistance during the experiments.

\section{Funding}

The present study was supported by Beijing Cancer Hospital Fund (grant no. 10-02).

\section{Availability of data and materials}

All data generated or analyzed during this study are included in this published article.

\section{Authors' contributions}

WY performed the experiments and wrote the manuscript. JS and NZ analyzed and interpreted the patient data. GX was a major contributor in the design of the study, and writing of the manuscript.

\section{Ethics approval and consent to participate}

Ethical approval was obtained from the Ethics Committee of Beijing University Cancer Hospital prior to these procedures, and all patients provided informed consent for inclusion in the present study.

\section{Consent for publication}

All patients provided informed consent for inclusion in the present study, and provided consent for publication.

\section{Competing interests}

The authors declare that they have no competing interests.

\section{References}

1. Siegel R, Ma J, Zou Z and Jemal A: Cancer statistics, 2014. CA Cancer J Clin 64: 9-29, 2014.

2. Chen AY, Jemal A and Ward EM: Increasing incidence of differentiated thyroid cancer in the United States, 1988-2005. Cancer 115: 3801-3807, 2009.

3. Cooper DS, Doherty GM, Haugen BR, Kloos RT, Lee SL, Mandel SJ, Mazzaferri EL, McIver B, Sherman SI and Tuttle RM; American Thyroid Association Guidelines Taskforce: Management guidelines for patients with thyroid nodules and differentiated thyroid cancer. Thyroid 16: 109-142, 2006.

4. Shaha AR, Shah JP and Loree TR: Patterns of nodal and distant metastasis based on histologic varieties in differentiated carcinoma of the thyroid. Am J Surg 172: 692-694, 1996.

5. Hay ID, Thompson GB, Grant CS, Bergstralh EJ, Dvorak CE, Gorman CA, Maurer MS, McIver B, Mullan BP, Oberg AL, et al: Papillary thyroid carcinoma managed at the Mayo Clinic during six decades (1940-1999): Temporal trends in initial therapy and long-term outcome in 2444 consecutively treated patients. World J Surg 26: 879-885, 2002.

6. Wong KP and Lang BH: The role of prophylactic central neck dissection in differentiated thyroid carcinoma: Issues and controversies. J Oncol 2011: 127929, 2011.

7. Keum HS, Ji YB, Kim JM, Jeong JH, Choi WH, Ahn YH and Tae K: Optimal surgical extent of lateral and central neck dissection for papillary thyroid carcinoma located in one lobe with clinical lateral lymph node metastasis. World J Surg Oncol 10: 221, 2012

8. Sadowski BM, Snyder SK and Lairmore TC: Routine bilateral central lymph node clearance for papillary thyroid cancer. Surgery 146: 696-705, 2009.

9. Carling T, Long WD III and Udelsman R: Controversy surrounding the role for routine central lymph node dissection for differentiated thyroid cancer. Curr Opin Oncol 22: 30-34, 2010.

10. Shaha AR: Prophylactic central compartment dissection in thyroid cancer: A new avenue of debate. Surgery 146: 1224-1227, 2009.

11. Clark OH: Thyroid cancer and lymph node metastases. J Surg Oncol 103: 615-618, 2011 
12. Kouvaraki MA, Shapiro SE, Fornage BD, Edeiken-Monro BS, Sherman SI, Vassilopoulou-Sellin R, Lee JE and Evans DB: Role of preoperative ultrasonography in the surgical management of patients with thyroid cancer. Surgery 134: 946-955, 2003.

13. Besic N, Pilko G, Petric R, Hocevar M and Zgajnar J: Papillary thyroid microcarcinoma: Prognostic factors and treatment J Surg Oncol 97: 221-225, 2008.

14. Tisell LE, Nilsson B, Mölne J, Hansson G, Fjälling M, Jansson S and Wingren U: Improved survival of patients with papillary thyroid cancer after surgical microdissection. World J Surg 20 : 854-859, 1996

15. Lundgren CI, Hall P, Dickman PW and Zedenius J: Clinically significant prognostic factors for differentiated thyroid carcinoma: A population-based, nested case-control study. Cancer 106: 524-531, 2006.

16. Hughes DT, White ML, Miller BS, Gauger PG, Burney RE and Doherty GM: Influence of prophylactic central lymph node dissection on postoperative thyroglobulin levels and radioiodine treatment in papillary thyroid cancer. Surgery 148: 1100-1107, 2010.

17. Kim WW, Park HY and Jung JH: Surgical extent of central lymph node dissection in clinically node-negative papillary thyroid cancer. Head Neck 35: 1616-1620, 2013.

18. Takami H, Ito Y, Okamoto T and Yoshida A: Therapeutic strategy for differentiated thyroid carcinoma in Japan based on a newly established guideline managed by Japanese Society of Thyroid Surgeons and Japanese Association of Endocrine Surgeons. World J Surg 35: 111-121, 2011.

19. Roh JL, Kim JM and Park CI: Central lymph node metastasis of unilateral papillary thyroid carcinoma: Patterns and factors predictive of nodal metastasis, morbidity, and recurrence. Ann Surg Oncol 18: 2245-2250, 2011.

20. Zhu Y, Chen X, Zhang H, Chen L, Zhou S, Wu K, Wang Z, Kong $\mathrm{L}$ and Zhuang H: Carbon nanoparticle-guided centra lymph node dissection in clinically node-negative patients with papillary thyroid carcinoma. Head Neck 38: 840-845, 2016.

21. Catarci M, Guadagni S, Zaraca F, Pistoia MA, Mastracchio A, Trecca A, Ruco L and Carboni M: Prospective randomized evaluation of preoperative endoscopic vital staining using $\mathrm{CH}-40$ for lymph node dissection in gastric cancer. Ann Surg Oncol 5: 580-584, 1998.

22. Cai HK, He HF, Tian W, Zhou MQ, Hu Y and Deng YC: Colorectal cancer lymph node staining by activated carbon nanoparticles suspension in vivo or methylene blue in vitro. World J Gastroenterol 18: 6148-6154, 2012.

23. Conti de Freitas LC, Phelan E, Liu L, Gardecki J, Namati E, Warger WC, Tearney GJ and Randolph GW: Optical coherence tomography imaging during thyroid and parathyroid surgery: A novel system of tissue identification and differentiation to obviate tissue resection and frozen section. Head Neck 36: 1329-1334 2014.

24. Ladurner R, Hallfeldt KK, Al Arabi N, Stepp H, Mueller S and Gallwas JK: Optical coherence tomography as a method to identify parathyroid glands. Lasers Surg Med 45: 654-659, 2013.
25. Glover AR, Gundara JS, Norlén O, Lee JC and Sidhu SB: The pros and cons of prophylactic central neck dissection in papillary thyroid carcinoma. Gland Surg 2: 196-205, 2013.

26. Kihara M, Miyauchi A, Kontani K, Yamauchi A and Yokomise H: Recovery of parathyroid function after total thyroidectomy: Long-term follow-up study. ANZ J Surg 75: 532-536, 2005.

27. Lee YJ, Kim DW, Park HK, Kim DH, Jung SJ, Oh M and Bae SK: Pre-operative ultrasound diagnosis of nodal metastasis in papillary thyroid carcinoma patients according to nodal compartment. Ultrasound Med Biol 41: 1294-1300, 2015.

28. Mulla M and Schulte KM: Central cervical lymph node metastases in papillary thyroid cancer: A systematic review of imaging-guided and prophylactic removal of the central compartment. Clinical Endocrinol (Oxf) 76: 131-136, 2012.

29. Kim BS, Ryu HS and Kang KH: The value of preoperative PET-CT in papillary thyroid cancer. J Int Med Res 41: 445-456, 2013.

30. Feng J and Wang J: Diagnosis and treatment of thyroid microcarcinoma. Lin Chuang Er Bi Yan Hou Tou Jing Wai Ke Za Zhi 28 1911-1917, 2014 (In Chinese)

31. Machens A, Holzhausen HJ and Dralle H: Skip metastases in thyroid cancer leaping the central lymph node compartment. Arch Surg 139: 43-45, 2004.

32. Ito $\mathrm{Y}$, Jikuzono $\mathrm{T}$, Higashiyama $\mathrm{T}$, Asahi S, Tomoda $\mathrm{C}$, Takamura Y, Miya A, Kobayashi K, Matsuzuka F, Kuma K and Miyauchi A: Clinical significance of lymph node metastasis of thyroid papillary carcinoma located in one lobe. World J Surg 30: 1821-1828, 2006

33. Lim YC, Choi EC, Yoon YH, Kim EH and Koo BS: Central lymph node metastases in unilateral papillary thyroid microcarcinoma. Br J Surg 96: 253-257, 2009.

34. Lee KE, Chung IY, Kang E, Koo do H, Kim KH, Kim SW, Youn YK and Oh SK: Ipsilateral and contralateral central lymph node metastasis in papillary thyroid cancer: Patterns and predictive factors of nodal metastasis. Head Neck 35: 672-676, 2013.

35. Mamelle E, Borget I, Leboulleux S, Mirghani H, Suárez C, Pellitteri PK, Shaha AR, Hamoir M, Robbins KT, Khafif A, et al: Impact of prophylactic central neck dissection on oncologic outcomes of papillary thyroid carcinoma: A review. Eur Arch Otorhinolaryngol 272: 1577-1586, 2015

36. Raffaelli M, De Crea C, Sessa L, Fadda G, Bellantone C and Lombardi CP: Ipsilateral central neck dissection plus frozen section examination versus prophylactic bilateral central neck dissection in cN0 papillary thyroid carcinoma. Ann Surg Oncol 22: 2302-2308, 2015

37. Hao RT, Chen J, Zhao LH, Liu C, Wang OC, Huang GL, Zhang XH and Zhao J: Sentinel lymph node biopsy using carbon nanoparticles for Chinese patients with papillary thyroid microcarcinoma. Eur J Surg Oncol 38: 718-724, 2012.

38. Sun SP, Zhang Y, Cui ZQ, Chen Q, Zhang W, Zhou CX, Xie PP and Liu BG: Clinical application of carbon nanoparticle lymph node tracer in the VI region lymph node dissection of differentiated thyroid cancer. Genet Mol Res 13: 3432-3437, 2014.

39. Yu W, Wang T and Zhang N: Parathyroid protection in surgery of bilateral papillary thyroid cancer. Zhonghua Er Bi Yan Hou Tou Jing Wai Ke Za Zhi 50: 406-410, 2015 (In Chinese). 